\title{
Vaping Induced Cardiac Complications - A Smokescreen Coming to Engulf the Heart?
}

\author{
Benji LIM*, MB BCh BAO, Sheldon LEE, MBBS and Siang Chew CHAI, MBBS
}

Department of Cardiology, Changi General Hospital, Singapore

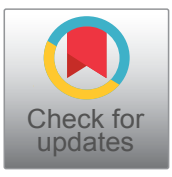

*Corresponding author: Benji Lim, MB BCh BAO, Department of Cardiology, Changi General Hospital, 2 Simei Street 3, Singapore 529889, Singapore, Tel: +65-69365626, Fax: $+65-62830387$

\begin{abstract}
E-cigarette usage has been shown to be associated with lung injury. However, there has been limited data of cardiovascular complications in e-cigarette users. We present two patients that developed cardiac complications after recent increased e-cigarette usage - the first with non-ischemic cardiomyopathy after a month of electronic cigarette usage at the highest power setting of 40 Watts, and the second with predominantly non-ischemic cardiomyopathy with underlying single vessel coronary artery disease after a threefold increase in e-cigarette usage for the preceding three months. In addition, to the best of our knowledge, this is the first report of an association between e-cigarette usage and heart failure.
\end{abstract}

\section{Keywords}

Smoking, E-cigarettes, Cardiomyopathy, Atrial fibrillation, Ischemic heart disease

\section{Case 1}

A 42-year-old Eurasian male with a background history of hypertension, diagnosed one and a half years prior to admission, and dyslipidemia presented with a five day history of exertional chest pain, dyspnea, orthopnea and leg swelling. He had no family history of cardiac disease and was a chronic smoker. Of note, he had started using e-cigarettes one month prior to admission at the highest power setting of 40 Watts.

On admission, his blood pressure was 158/125 $\mathrm{mmHg}$, heart rate 80 beats per minute $(\mathrm{bpm})$, temperature $37.6{ }^{\circ} \mathrm{C}$ and oxygen saturation $100 \%$ on room air. On examination, no murmurs were detected but there were bibasal lung crepitations and bilateral lower limb pitting edema.
Serial cardiac enzymes, full blood count, inflammatory markers, renal, thyroid and liver function tests were within normal limits. NT-proBNP was elevated $(14,746$ $\mathrm{pg} / \mathrm{ml}$ ). A chest radiograph showed cardiomegaly and pulmonary congestion while an electrocardiogram (ECG) showed sinus rhythm, left ventricular hypertrophy and widespread T wave inversions (Figure 1).

A transthoracic echocardiogram (TTE) showed a severely impaired left ventricular ejection fraction (LVEF) of $20-25 \%$ with global hypokinesia. Importantly, there were no features of left ventricular hypertrophy (end-diastolic interventricular septum thickness 7.76 $\mathrm{mm}$, end-diastolic left ventricular posterior wall thickness $9.52 \mathrm{~mm}$, indexed left ventricular mass $95.6 \mathrm{~g} / \mathrm{m}^{2}$ ) or any significant valvular abnormality. Coronary angiography showed luminal irregularities in all three coronary arteries (Figure 2-4).

Magnetic resonance imaging of the heart showed a dilated and diffusely hypokinetic left ventricle with an LVEF of $24 \%$ and late gadolinium enhancement in the basal septal midwall, suggesting an underlying dilated, non-ischemic cardiomyopathy.

The patient was administered intravenous diuretics with prompt resolution of symptoms. Concurrently, he was prescribed guideline-directed management and therapy consisting of an angiotensin receptor-neprilysin inhibitor, beta-blocker, ivabradine and an aldosterone antagonist, in addition to a loop diuretic and statin. He was also advised regarding smoking cessation and fluid restriction. A repeat TTE is planned for in the near future to reassess his LVEF. 


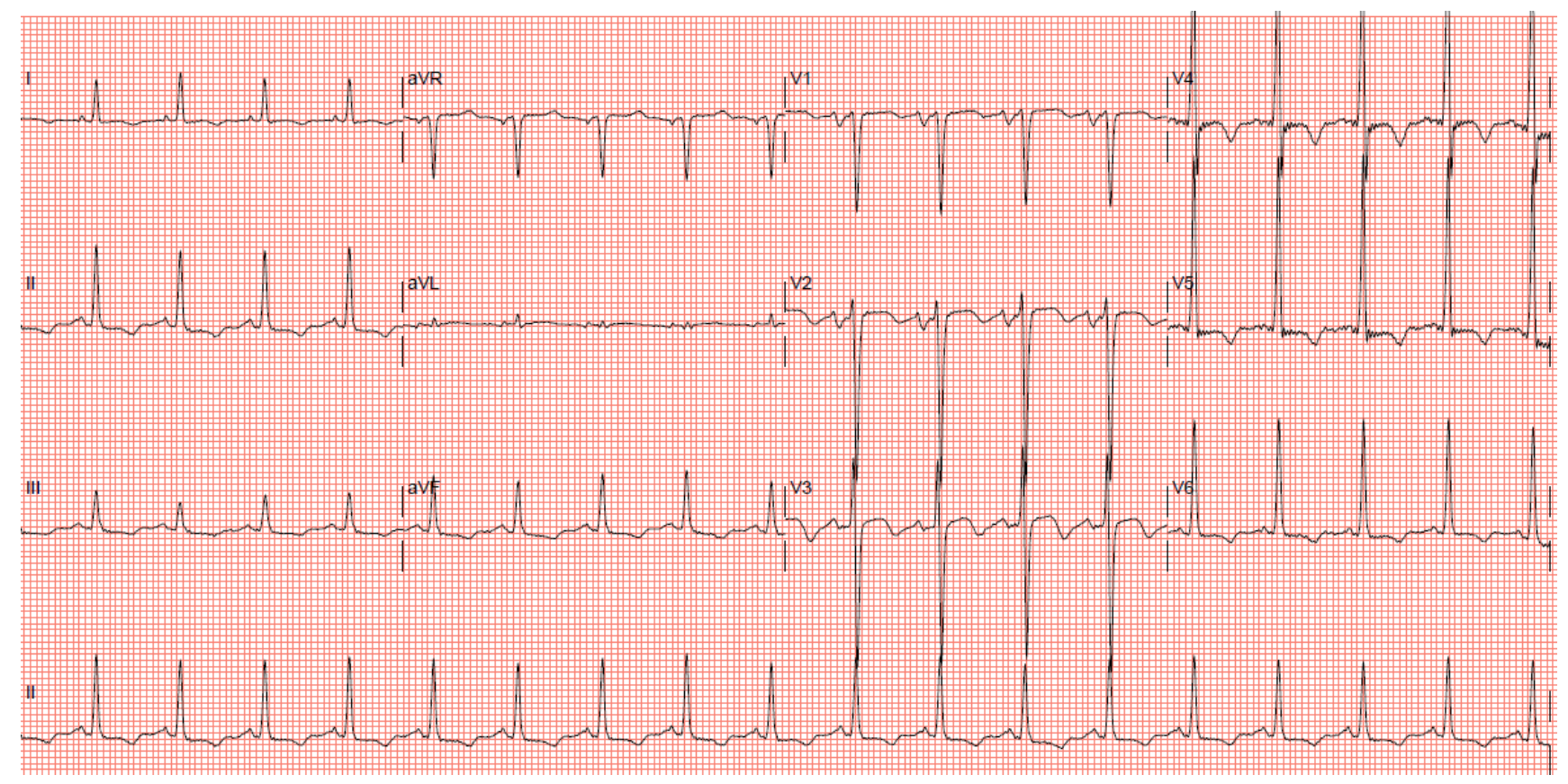

Figure 1: Electrocardiogram showing sinus rhythm, left ventricular hypertrophy and widespread T wave inversions.

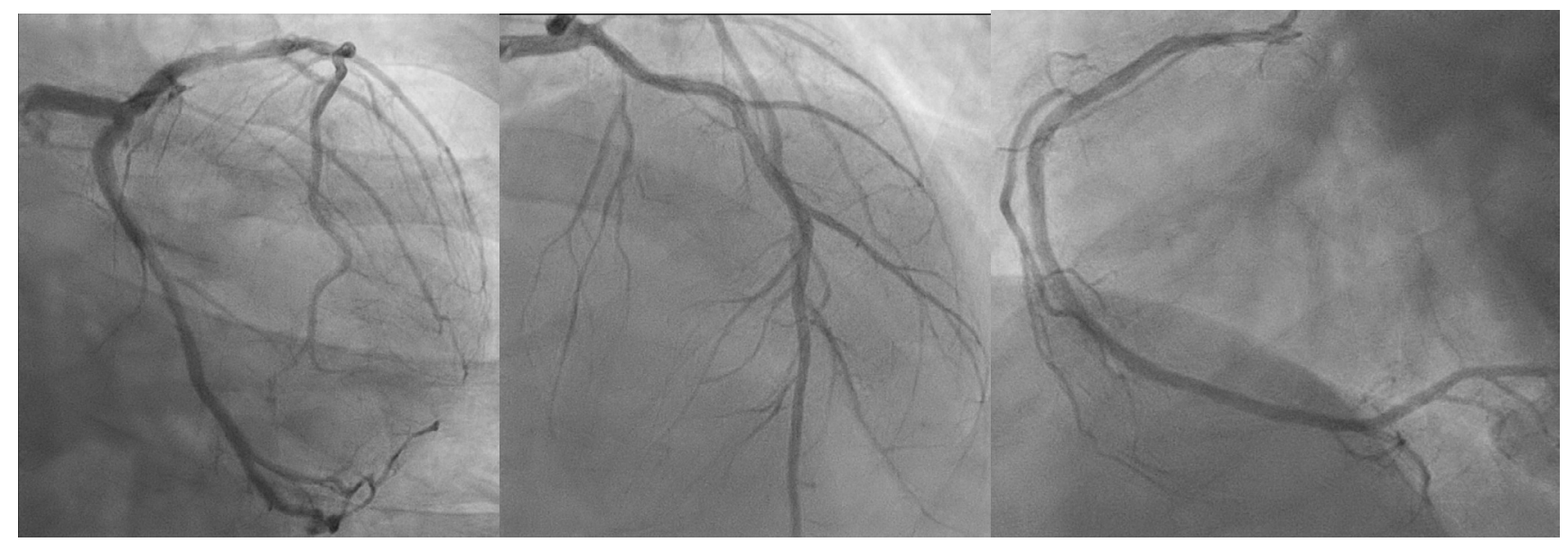

Figures 2-4: Coronary angiogram showing luminal irregularities in all 3 coronary arteries. From left to right: AP-caudal view showing the left circumflex artery, AP-cranial view showing the left anterior descending artery, LAO view showing the right coronary artery.

\section{Case 2}

A 52-year-old Chinese male with a background history of diabetes mellitus, hypertension and dyslipidemia presented with a one week history of abdominal discomfort, bloatedness and constipation. He had a mild cough for three days and developed dyspnea and palpitations on the day of admission. Two years prior to admission, a myocardial perfusion scan performed for exertional dyspnea was negative for ischemia with an LVEF of $55 \%$. He had no family history of cardiac disease and was a chronic smoker. He had previously been using one cartridge every three days in his e-cigarette, but in the preceding three months, he had tripled his e-cigarette usage to one cartridge per day. He also drank four bottles of beer per day for the past thirty years.

On admission, his blood pressure was $128 / 93 \mathrm{mmHg}$, heart rate $214 \mathrm{bpm}$, temperature $36.7^{\circ} \mathrm{C}$ and oxygen saturation $98 \%$ on room air. On examination, no murmurs were detected, lungs were clear, abdomen was distended but soft and non-tender with normal bowel sounds and there was no lower limb edema.

Serial Troponin-T $(32,35$ and $28 \mathrm{ng} / \mathrm{L}$ - normal range $<30 \mathrm{ng} / \mathrm{L})$ and NT-proBNP $(2,594 \mathrm{pg} / \mathrm{ml})$ were elevated. Hemoglobin, inflammatory markers, electrolytes, thyroid and liver function tests were normal. White blood cell count was slightly elevated at $12.8 \times 10^{3} / \mathrm{uL}$, but C-reactive protein and procalcitonin were normal (3.7 $\mathrm{mg} / \mathrm{L}$ and $0.22 \mathrm{ug} / \mathrm{L}$ respectively). However, serum creatinine was acutely elevated ( $174 \mathrm{umol} / \mathrm{L}$ compared to $109 \mathrm{umol} / \mathrm{L}$ just three months prior) and lactate was abnormally high $(6.7 \mathrm{mmol} / \mathrm{L})$. An arterial blood gas showed metabolic acidosis with respiratory compensation $\left(\mathrm{pH}\right.$ 7.050, $\mathrm{pO}_{2} 138 \mathrm{mmHg}, \mathrm{pCO}_{2} 41 \mathrm{mmHg}$, 


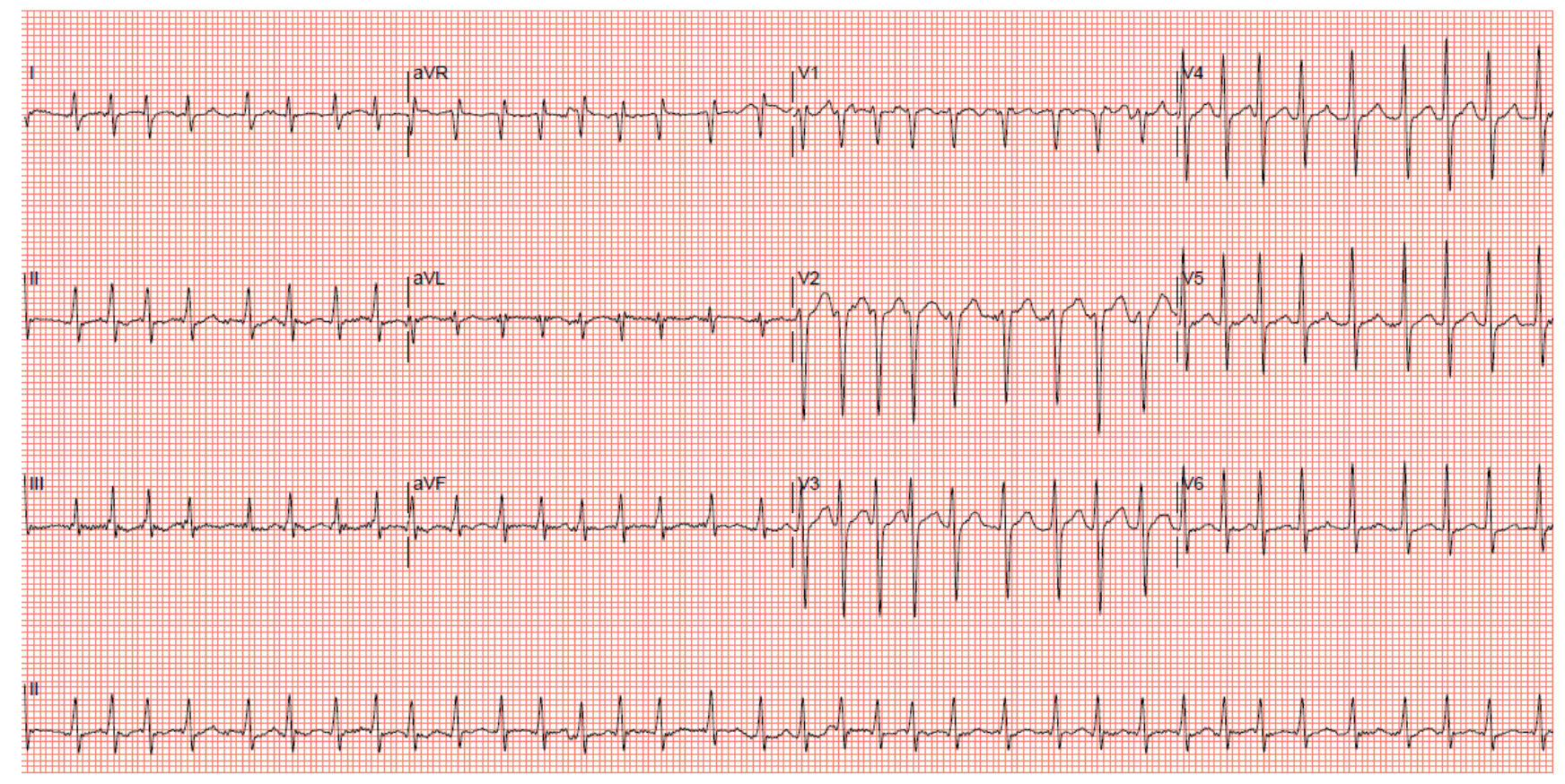

Figure 5: Electrocardiogram showing atrial fibrillation with a ventricular rate of 218 beats per minute.

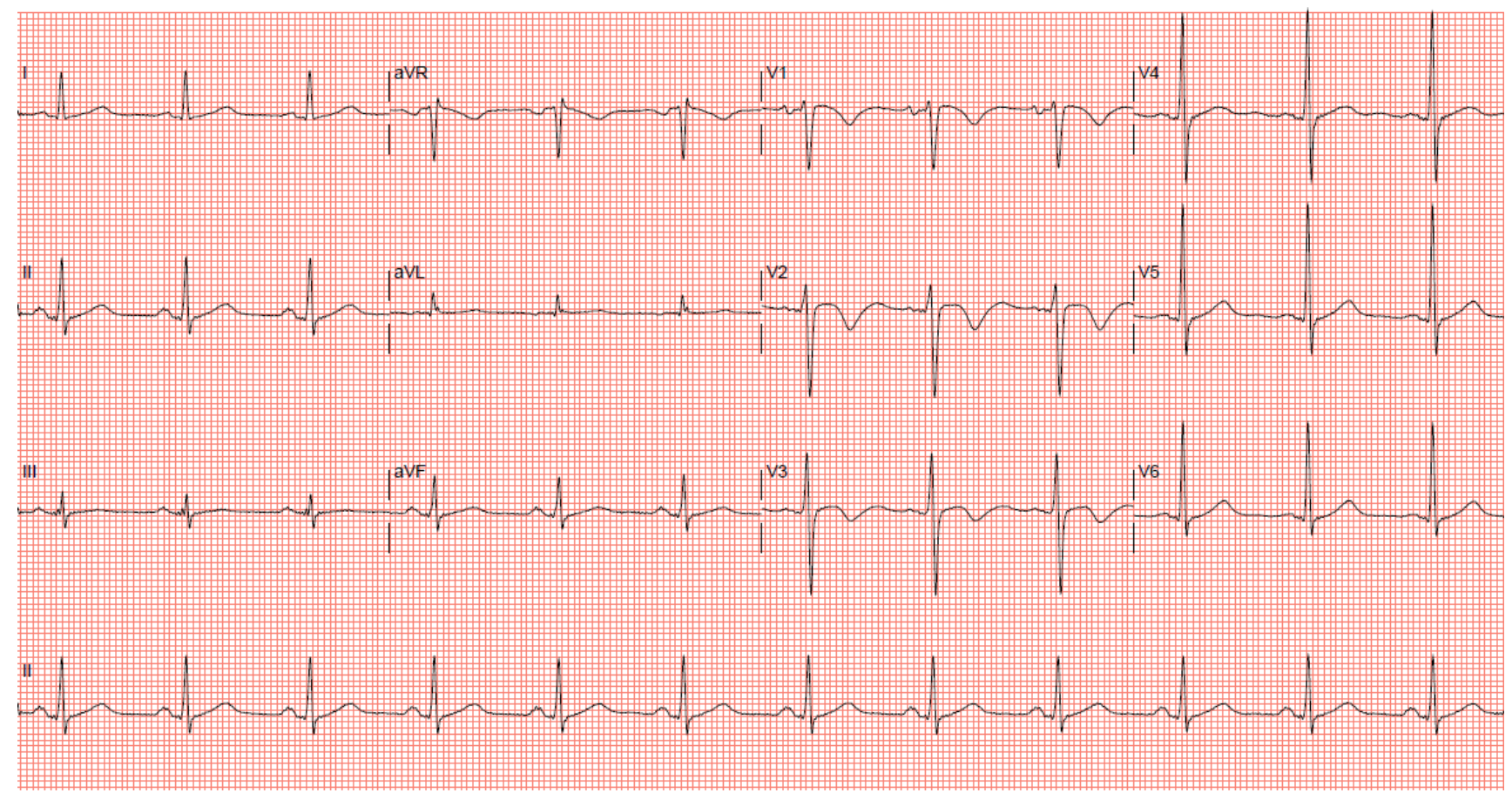

Figure 6: Electrocardiogram showing conversion to sinus rhythm with T wave inversions in leads V1-3.

bicarbonate $11 \mathrm{mmol} / \mathrm{L})$. A chest radiograph showed cardiomegaly and pulmonary congestion while an ECG showed atrial fibrillation (AF) with a rapid ventricular rate of $218 \mathrm{bpm}$ (Figure 5).

The patient was administered intravenous amiodarone infusion but required intubation shortly after admission for respiratory distress secondary to acute pulmonary edema. Intravenous diuretics were administered with good negative fluid balance and resolution of the chest radiograph changes within the first few days.

An urgent TTE on the day of admission during AF with a rapid ventricular rate of 138 beats per minute showed a non-dilated left ventricle with an LVEF of $10-15 \%$ with global hypokinesia. Myocardial thickness remained preserved with no scarring. The left and right ventricles and left atrium were of normal size but the right atrium was mildly dilated (end-diastolic LV internal diameter 50.8 $\mathrm{mm}$, indexed LA volume $21.15 \mathrm{ml} / \mathrm{m}^{2}$, RA area $19.3 \mathrm{~cm}^{2}$ ). There was mild mitral regurgitation, moderate to severe tricuspid regurgitation due to a dilated tricuspid annulus $(31.9 \mathrm{~mm})$ and the estimated right ventricular systolic pressure (RVSP) was $38 \mathrm{mmHg}$. A transesophageal echocardiogram ruled out intracardiac thrombi but de- 
tected an incidental small inferior sinus venosus ASD of $0.6 \mathrm{~cm}$ with predominantly left to right shunting on color Doppler. In view of his presenting abdominal symptoms, elevated lactate and AF, a computed tomography mesenteric angiogram was performed which ruled out ischemic bowel and did not detect any septic sources in the abdomen or visualized lung bases.

Amiodarone was stopped the next day because of severe transaminitis. Despite maximally tolerated doses of digoxin and bisoprolol, his heart rate remained at $130-160 \mathrm{bpm}$ for the next five days. Synchronized direct current cardioversion (DCCV) was performed on days one and four of admission but with only transient conversion to sinus rhythm, each lasting less than thirty seconds. The patient's heart rate eventually slowed down after one week, and spontaneously converted to sinus rhythm on day eight (Figure 6).

Coronary angiography on day ten of admission showed diffuse $50-65 \%$ stenosis in the ostial to mid LAD artery, luminal irregularities in the left circumflex artery, and $30 \%$ stenosis in the mid right coronary artery (Figure 7-10). Fractional flow reserve (FFR) measurement in the LAD was 0.72 and two overlapping drug-eluting stents (DES) were deployed in the ostial to mid LAD. Post-percutaneous coronary intervention (PCI) FFR was 0.80 . However, this degree of coronary artery disease
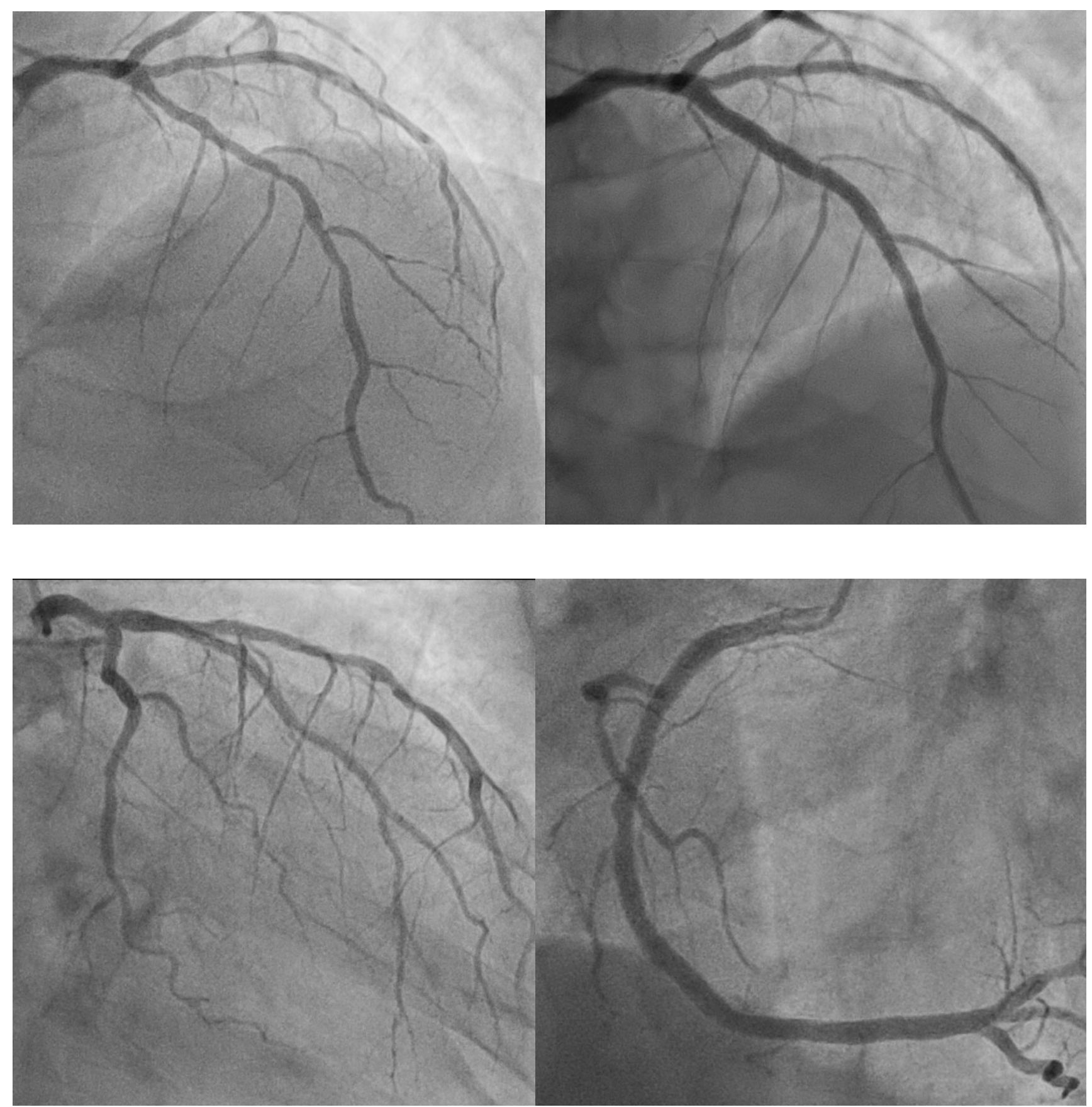

Figures 7-10: Coronary angiogram. Top left (AP-cranial view): Moderate stenosis from the ostium to mid left anterior descending artery (LAD); Top right (AP-cranial view): LAD post-stenting; Bottom left (RAO-caudal view): Left circumflex artery with luminal irregularities; Bottom right (LAO view): $30 \%$ stenosis in the mid right coronary artery. 
alone would not have been able to account for such severely impaired LVEF.

In addition to triple anti-thrombotic therapy post$\mathrm{PCl}$ and high intensity statin, the patient was prescribed guideline-directed management and therapy consisting of an angiotensin-converting enzyme inhibitor, beta-blocker and aldosterone antagonist. He was also advised regarding smoking cessation and fluid restriction.

A follow-up TTE performed 7 months later when the heart rate was $87 \mathrm{bpm}$ in AF showed an LVEF of $20 \%$. The rest of the TTE findings were unchanged from the previous TTE except for the estimated RVSP which had come down to $24 \mathrm{mmHg}$, likely as a result of adequate diuresis and the patient being euvolemic at that time.

\section{Discussion}

Since mid-2019, reports of e-cigarette or vaping product use associated lung injury (EVALI) started emerging in the United States among users of e-cigarettes [1]. As of February 18, 2020, a total of 2,807 hospitalized EVALI cases have been reported by the U.S. Centers for Disease Control, amongst which 68 deaths have been confirmed [2]. Investigators have identified vitamin $\mathrm{E}$ acetate, an additive in some hydrocannabinol (THC) containing e-cigarette products, to be strongly associated with the EVALI outbreak [3].

Although two retrospective studies showed an increased risk of stroke [4] and myocardial infarctions [5], there has generally been a lack of evidence from clinical trials or long-term cohort studies regarding the cardiovascular effects and complications of e-cigarettes. Two knowledge gaps have been identified - prospective studies assessing the effects of e-cigarettes on clinical cardiovascular outcomes are lacking, and it is unknown whether different patterns of e-cigarette smoking (with respect to age of onset, frequency and cumulative duration of use) exert differential cardiovascular effects [6]. To the best of our knowledge, we present the first report of a possible association between recent increased e-cigarette usage and the development of acute decompensated heart failure and refractory AF.

Our first patient developed non-ischemic cardiomyopathy following a short period of high power e-cigarette usage. Although non-ischemic cardiomyopathy could have many etiologies, our investigations did not suggest other common causes such as hypertrophic, infectious or drug related cardiomyopathies. Dilated cardiomyopathy with a genetic component could be a differential in view of his young age, but there was no family history of cardiomyopathy or sudden death. Genetic and family counseling might be warranted if his heart function remained poor despite a trial of optimal medical therapy for at least three months. Another etiology in view of his history of hypertension would be hypertensive heart disease. However, there were no features of left ventricular hypertrophy on echocardiog- raphy or magnetic resonance imaging and he was only diagnosed with hypertension one and a half years prior to admission which would make rapid progression to a burnt-out hypertensive heart disease state less likely.

Our second patient presented with congestive cardiac failure secondary to a predominantly non-ischemic cardiomyopathy with underlying single vessel coronary artery disease following a short period of increased e-cigarette usage. The patient had multiple other risk factors for atherosclerotic heart disease (diabetes, hypertension, dyslipidemia and smoking) and cardiomyopathy (chronic alcohol intake and AF with rapid ventricular rate) and hence, it would be unlikely that the increased e-cigarette usage alone would have accounted for his condition. However, the normal left atrium and ventricle size would suggest that the AF was of recent onset and that tachycardia induced cardiomyopathy would be a less likely differential.

It was striking how extremely rapid our second patient's ventricular rate in AF was, which was also refractory to anti-arrhythmic and rate controlling agents as well assynchronized DCCV attempts. AF with rapid ventricular rate would usually be secondary to other causes such as sepsis, blood loss or dehydration. Though the patient did present with a mild cough, this was attributed to the pulmonary edema on admission. There were no other localizing symptoms suggestive of infection and he was afebrile. There had been no recent overseas travel or sick contacts. His initial blood works on admission revealed mild leukocytosis but infective markers were bland. The chest radiograph had shown bilateral infiltrates which resolved within the first few days with diuresis and hence, the overall picture was more in keeping with acute pulmonary edema rather than pneumonia. Furthermore, a computed tomography mesenteric angiogram did not detect any foci of infection in the abdomen or visualized lung bases. In addition, he was never anemic or dehydrated during the admission. A cardiac magnetic resonance imaging would have been useful to ascertain the etiology of his cardiomyopathy; however, in view of the risk of nephrogenic systemic fibrosis due to his impaired renal function, this was not performed.

We recognize that that the post-PCI FFR of 0.80 in our second patient was borderline ischemic. We had implanted 2 overlapping DES $(3.0 \times 38 \mathrm{~mm}$ and a $3.0 \times 18$ $\mathrm{mm}$ ) that were post-dilated with a $3.5 \mathrm{~mm}$ non-compliant balloon to $17 \mathrm{~atm}$. On the final angiographic result in orthogonal views, there was a small step-down at the distal stent edge. Although we cannot fully exclude stent under-expansion as we do not routinely utilize IVUS or OCT for imaging due to reimbursement reasons, however, available literature indicates that diffuse disease [7], LAD lesions [7,8] and total stent length exceeding $50 \mathrm{~mm}$ [9] are associated with a suboptimal post- $\mathrm{PCl}$ FFR result of $<0.80$. 
In both cases, our patients developed cardiovascular conditions after a recent history of high intensity e-cigarette usage. It has been shown that the primary toxicants in e-cigarettes emissions are acrolein, formaldehyde and acetaldehyde which are generated in larger amounts when the e-cigarettes are used at higher power settings $[10,11]$.

Because of limited clinical studies evaluating the effects of these toxicants in humans, most evidence come from animal studies. Acrolein has toxic effects on cardiac myocytes due to the direct acrolein-protein adduct formation in cardiac mitochondria which can limit adenosine triphosphate formation [12]. It can also provoke oxidative stress in cardiac myocytes and inhibit glutathione S-transferase leading to depletion of the intracellular anti-oxidant glutathione, thus increasing myocyte susceptibility to free radical stress [13]. Similarly, aldehydes are associated with oxidative stress and myocyte dysfunction [14]. These effects contribute to myocyte necrosis, dysfunction and ultimately cardiomyopathy [15]. Acrolein can also activate TRPA1 (transient receptor potential cation channel, subfamily $A$, member 1 ) which results in autonomic imbalance, thus increasing the risk of ventricular arrhythmias [16].

Traditional tobacco cigarettes have been linked to the development of AF [17], possibly due to increased sympathetic nervous activity. Similarly, e-cigarettes have been shown to produce abnormal patterns of heart rate variability as reported in traditional tobacco cigarette users consistent with activation of the sympathetic nervous system [18]. Therefore, it is likely that e-cigarette usage, particularly at higher power settings, results in AF with higher ventricular rates and possibly more refractory to conventional anti-arrhythmic strategies.

\section{Conclusion}

This case series provides further evidence of links between e-cigarette usage and cardiac complications. In future, doctors may need to take a detailed e-cigarette usage history which includes size of cartridges used, power setting, and whether there has been recent increased usage. In addition, association between increased e-cigarette usage and heart failure or refractory AF has not previously been described. More studies would be necessary to definitively link a causal relationship between the two. This case series would hopefully be the first step towards this goal.

\section{Learning Objectives}

To highlight possible cardiac complications associated with e-cigarette usage.

To highlight the importance of a detailed e-cigarette smoking history which includes size of cartridges used, power setting, and whether there has been recent increased usage.

\section{Acknowledgements}

The authors have no conflicts of interest to declare and received no financial support in the writing of this article.

\section{Funding}

No funding supported the writing of this publication.

All authors contributed equally to the publication of this article.

\section{References}

1. Layden JE, Ghinai I, Pray I, Kimball A, Layer M, et al. (2020) Pulmonary illness related to e-cigarette use in Illinois and Wisconsin - Final report. N Engl J Med 382: 903-916.

2. Centers for Disease Control and Prevention (2020) Outbreak of lung injury associated with E-cigarette use, or vaping, products.

3. Blount BC, Karwowski MP, Shields PG, Morel-Espinosa M, Valentin-Blasini L, et al. (2020) Vitamin E acetate in bronchoalveolar-lavage fluid associated with EVALI. N Engl J Med 382: 697-705.

4. Ndunda PM, Muutu TM (2019) Electronic cigarette use is associated with a higher risk of stroke. Stroke 50: A9.

5. Alzahrani T, Pena I, Temesgen N, Glantz SA (2018) Association between electronic cigarette use and myocardial infarction. Am J Prev Med 55: 455-461.

6. Kavousi M, Pisinger C, Barthelemy J-C, Smedt DD, Koskinas K, et al. (2020) Electronic cigarettes and health with special focus on cardiovascular effects: Position paper of the European Association of Preventive Cardiology (EAPC). Eur J Prev Cardiol.

7. Agarwal SK, Kasula S, Almomani A, Hacioglu Y, Ahmed Z, et al. (2017) Clinical and angiographic predictors of persistently ischemic fractional flow reserve after percutaneous revascularization. Am Heart J 184: 10-16.

8. Kimura Y, Tanaka N, Okura H, Yoshida K, Akabane M, et al. (2016) Characterization of real-world patients with low fractional flow reserve immediately after drug-eluting stents implantation. Cardiovasc Interv Ther 31: 29-37.

9. Baranauskas A, Peace A, Kibarskis A, Shannon J, Abraitis $\mathrm{V}$, et al. (2016) FFR result post $\mathrm{PCl}$ is suboptimal in long diffuse coronary artery disease. Eurointervention 12: 14731480.

10. Benowitz NL, Fraiman JB (2017) Cardiovascular effects of electronic cigarettes. Nat Rev Cardiol 14: 447-456.

11. Qasim H, Karim ZA, Rivera JO, Khasawneh FT, Alshbool FZ (2017) Impact of electronic cigarettes on the cardiovascular system. J Am Heart Assoc 6.

12. Wang G, Guo Y, Vondriska TM, Zhang J, Zhang S, et al. (2008) Acrolein consumption exacerbates myocardial ischemic injury and blocks nitric oxide-induced PKCe signaling and cardioprotection. Journal of Molecular and Cellular Cardiology 44: 1016-1022.

13. Kehrer JP, Biswal SS (2000) The molecular effects of acrolein. Toxicol Sci 57: 6-15.

14. Güleç M, Songur A, Sahin S, Ozen OA, Sarsilmaz M, et al. (2006) Antioxidant enzyme activities and lipid peroxidation products in heart tissue of subacute and subchronic formaldehyde-exposed rats: A preliminary study. Toxicol Ind Health 22: 117-124. 
15. Ismahil MA, Hamid T, Haberzettl P, Gu Y, Chandrasekar B, et al. (2011) Chronic oral exposure to the aldehyde pollutant acrolein induces dilated cardiomyopathy. Am J Physiol Heart Circ Physiol 301: 2050-2060.

16. Hazari MS, Haykal-Coates N, Winsett DW, Krantz QT, King C, et al. (2011) TRPA1 and sympathetic activation contribute to increased risk of triggered cardiac arrhythmias in hypertensive rats exposed to diesel exhaust. Environ Health Perspect 119: 951-957.
17. Heeringa J, Kors JA, Hofman A, van Rooij FJA, Witteman JCM (2008) Cigarette smoking and risk of atrial fibrillation: The Rotterdam Study. Am Heart J 156: 1163-1169.

18. Moheimani RS, Bhetraratana M, Yin F, Peters KM, Gornbein J, et al. (2017) Increased cardiac sympathetic activity and oxidative stress in habitual electronic cigarette users: Implications for cardiovascular risk. JAMA Cardiol 2: 278284. 